These factors have already proved important in the case of keratin?

(2) The intermolecular contractile factor due to a variation in the number of ionised groups belonging to single molecules. This factor, which has not yet made its appearance in the literature of protein chemistry, is nevertheless an obvious possibility. The number of ionised groups possessed by a nucleic acid molecule $\theta^{8}$ varies considerably with $p H$. The chromosome micelle-a protamine nucleate aggregate ${ }^{9}$ will therefore be capable of strikingly different configurations for different $p H$ values, the nearer the $p H$ approaches to the isoelectric point of the protamine nucleate the greater being the degree of contraction. In view of the differential density of nucleic acid along the length of the chromosome, differential condensation (heteropycnosis) ${ }^{4}$ is naturally to be expected. This type of contractile factor also offers a simple interpretation of the results lately obtained by Kuwada and Darlington with ammonia vapour and with acetic and nitric acid ${ }^{3}$.

(3) The intramolecular contractile factor as exemplified by keratin?. In the case of keratin a contractile factor of 2 in particular has been attributed to intramolecular folds of the nature of linked pseudo diketo piperazine rings. Evidently there may be other and more considerable degrees of contraction if the rings in question are formed not by two con. secutive amino acid residues but by a run of $3,6,7$, $10,11, \ldots, 4 n-2,4 n-1$ residues. The first of these, vielding a contractile factor rather greater than $8 / 3$, may be the explanation of the contractile factor of about 3 recently announced for myosin films ${ }^{10}$. I now suggest that the chromosome micelle, evidently less stable than myosin and considerably less stable than keratin, will prove capable of a grosser type of folding, which will yield a far higher factor than the 2 of hair and the 3 of myosin.

(4) The intramolecular contractile factors due to the sharing of an electron by the ketonic oxygen and the hydrogen atom belonging respectively to the carbon and nitrogen atoms in the backbone of a protamine molecule ${ }^{11}$.

(5) The macroscopic 'twizzling' factor as exemplified by keratin fibres ${ }^{12}$. This contractile factor is of quite different type from the three preceding ones. It is "macroscopic' in the sense that it is a property not of individual molecules but of the micelle as a whole.

Mathematical Institute,

\section{M. WRINCH.} Oxford.

April 6.

INATURE, 134, 978, December 22, 1934.

2 "Chromosomes and their Structure". (In course of publication in the J. Roy. Micro. Soc.)

${ }^{3} \mathrm{C}$. D. Darlington, "Recent Advances in Cytology". London, 1932. Y. Kuwada and T. Nakamura, Mem. Coll. Sci, Kyoto, 9, 129$139 ; 1933$. Y. Kuwada and T. Nakamura, ibid., 9, 343-366; 1934. J. Genet., 29, 159-173; 1934 .

ic. D. Darlington, Cutologia, 4, 229-240; 1933

s. S. Painter, J. Hered., 25, 465-476; 1934 . C. W. Metz and E. F. Say Proc. Nat. Acad. Sci. 20, $617-621$ 1934 R. L King and H. W. Beams, $J$. Morph., 56, 577-588; 1934. H. J. Muller and A Prokofleva, Comptes Rendus de l'Acad. des Sciences de l'U.R.S.S. 4 . 74-83, October 11, 1934. $\mathrm{P}$. Ch. Koller, NATURE, 135, 69, January 12, 1935 .

- Reference should be made to the literature of the "zwitterion", specially to $\mathrm{L}_{\text {. }}$ J. Harris, Biochem. J., 24, 1080-1097; 1930.

'W. T. Astbury and H. J. Woods, Phit. Trans. Roy. Soc., A, 232, $333-394 ; 1933$

Oxford, 1933. - D. Jordan Lloyd, J. Int. Soe. of Leather Trades' Chemists, 245$258 ; 1933$.

W. T. Astbury, NATURE, 135, 95, Jan. 19, 1935

11 D. Jordan Lloyd, Biol. Rev., 7, 254-273; 1932. 1934.

\section{$\alpha$-Tracks in Presence of Strong $\gamma$-Radiation}

IN investigations of nuclear transformations, it is sometimes desirable to be able to observe $\alpha$-particles (or protons) in the presence of strong $\gamma$-radiation. This has already been successfully accomplished by means of an ionisation chamber connected up to a valve-amplifier and oseillograph (as in the investiga. tions of Chadwick and Goldhaber ${ }^{1}$ on the photo. disintegration of heavy hydrogen).

It may be of interest that, under suitable conditions, it is also possible to observe $\alpha$-particles in the presence of strong $\gamma$-radiation by the Wilson cloud method. The accompanying photograph (Fig. 1) shows the tracks of $\alpha$-particles from polonium deposited on the outside of the brass cylinder $A$, in the presence of $\gamma$-rays from a $25 \mathrm{mgm}$. emanation tube placed inside $A$.

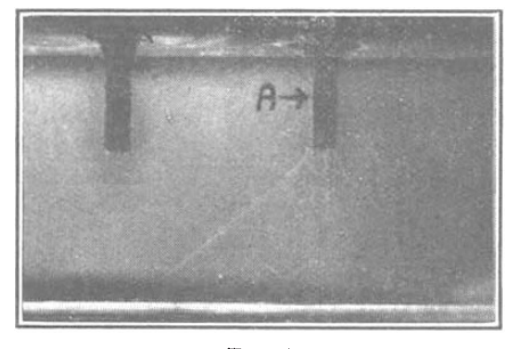

FIG. 1.

The best conditions appear to be obtained with hydrogen in the chamber, at an initial pressure of about $40 \mathrm{~cm}$. mercury, and a high expansion ratio somewhat above 1.4. Under these conditions, about 60 per cent of the $\alpha$-tracks observed in the absence of $\gamma$-radiation are still observed when a source of about $25 \mathrm{mgm}$. is placed in $A$. The conditions become rapidly worse if the strength of the source is increased much above $30 \mathrm{mgm}$. and this appears to be about the maximum unfiltered emanation source that can be placed inside the chamber without destroying the cloud tracks of $\alpha$-particles.

About five hundred photographs were taken with the cylinder $A$ covered with beryllium, and a 20-30 mgm. emanation source inside $A$, in order to investigate further the disintegration of beryllium by $\gamma$-rays, observed some time ago by Szilard and Chalmers ${ }^{2}$. No $\alpha$-tracks were observed. Assuming that tracks of range $R \mathrm{~cm}$. in air are actually produced, this means that the cross-section for the photo-disintegration of a Be nucleus is less than about $5 \times 10^{-30} R^{-1} \mathrm{~cm} .{ }^{2}$.

\section{Physical Laboratory, \\ University.}

D. Cameron.

Manchester.

IATURE, 134, $237 ; 1934$.
2 NATURE, 134, $494 ; 1934$.

\section{The Spectral Selective Photo-Electric Effect}

ZENER has recently ${ }^{1}$ discussed Fowler's theory ${ }^{2}$ of the spectral selective photo-electric effect, and concludes that it is incapable of explaining the observed order of magnitude of the emitted current. Inasmuch as a direct calculation ${ }^{3}$ of the photo-electric emission from a pure metal yields a spectral distribution curve 